The Office of AIDS Research, a relatively small but influential unit within the US National Institutes of Health, is responsible for "coordinating the scientific, budgetary, legislative, and policy elements of the NIH AIDS research program". Here the new director, Neal Nathanson, presents his vision of how the nations $\$ 1.7$ billion AIDS research effort should be used to respond to the challenge of AIDS.

\title{
Harnessing research to control AIDS
}

The Office of AIDS Research (OAR) is a unit of the National Institutes of Health (NIH), United States Department of Health and Human Services. The OAR is responsible for setting the scientific agenda for AIDS research at NIH, a large program that has grown over the past 15 years to a level of $\$ 1.7$ billion in the current fiscal year. It is OAR's mandate to plan, coordinate and determine the relative distribution of effort across a wide variety of research goals. To fulfill this responsibility, it is necessary to bring a broad vision to this very diverse research program. Periodically, it is useful to re-examine and articulate this vision, and the appointment of a new director of OAR provides an opportune moment for such a re-examination.

There are many perspectives that could shape the AIDS research program, but I have chosen to frame this discussion from the perspective of the epidemic itself, asking where and how research can intervene, providing the tools needed to reduce transmission and ameliorate the burden of disease. Thus I am proposing that we address our research agenda with a particular focus on the demographics of the epidemic and on "intervention research" strategies for the impacted populations. It is also important to note that, although the primary responsibility of the NIH is to fund the United States AIDS research program, our plans take cognizance of the global status of AIDS, since the dynamics of the epidemic are dramatically distinct in different countries. The exploding nature of the epidemic in those countries most severely affected has escalated the urgency of improved intervention strategies.

It is a truism that AIDS is the plague of the $20^{\text {th }}$ century, a disease that has already caused more than $10,000,000$ deaths since its appearance in the late $1970 \mathrm{~s}$. With an estimated $30,000,000$ current infections and new infections occurring at the rate of $>250,000$ monthly, the magnitude of the pandemic is difficult to comprehend. It is estimated that 2.3 million people worldwide died of AIDS in 1997-a 50 percent increase over 1996. Furthermore, more than 90 percent of cases occur in the poor-

est areas of the world that are the least equipped to control or treat a rampant and fatal new infectious disease.

How then can the extensive biomedical research resources of the United States be most effectively deployed to cope with this formidable challenge? Let us begin with a brief summary of the dynamics of the AIDS epidemic in the United States (where it has been well documented) (Fig. 1). Such an analysis provides potential points of intervention. Prevalence. In 1997, there were at least 650,000 HIV-infected individuals, of whom about 275,000 met the definition of AIDS. A substantial fraction of these HIV-infected persons are not receiving effective antiretroviral treatment, either because their infections have not been diagnosed, because they do not have access to adequate medical care or health insurance, or because they or their physicians have opted to defer treatment. Incidence. In the United States last year, there were approximately 40,000 new infec-

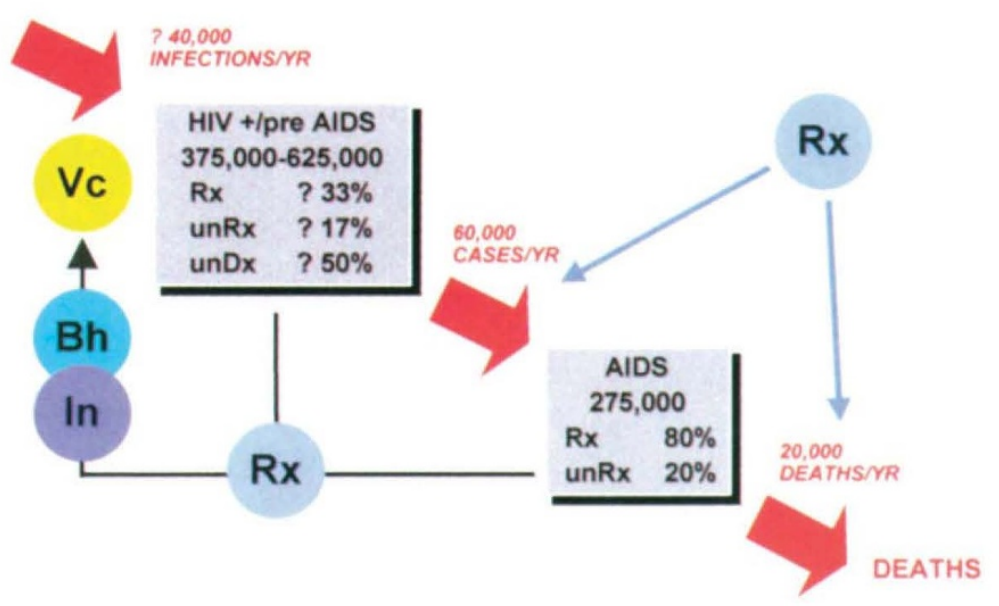

Fig. 1 Dynamics of the AIDS epidemic and points of intervention. Therapy (Rx) has reduced the annual incidence of new AIDS cases and the number of AIDS deaths. Transmission could be reduced by intervention at many levels, including antiviral therapy and treatment of sexually transmitted diseases $(\mathrm{Rx})$, microbicidal and other interventions (In), behavioral modification (Bh), and vaccination (VC). Parameters for the United States, 1997, estimated from data provided by CDC, USPHS. Numbers of new infections and numbers of patients undiagnosed, untreated, or treated are at best "guesstimates". UNITED STATES, 1997

tions, 60,000 new AIDS cases, and 20,000 deaths. Thus, we have three initial points for intervention in the epidemic: prevention of transmission; prevention of progression to disease; and prevention of mortality.

But in reality the epidemic consists of a number of quite distinct subepidemics. AIDS cases continue to rise dramatically among women, minorities, heterosexuals, adolescents, and drug users in our country. According to CDC, in 1996 new AIDS cases increased by $19 \%$ among heterosexual African American men; $12 \%$ among heterosexual African American women; $13 \%$ among Hispanic men; and 5\% among Hispanic women. Cases also increased in Native Americans, Asian Pacific Islanders, and other minority populations. Adolescents are particularly vulnerable to HIV infection as a result of several risk factors including high rates of unprotected intercourse, sexually transmitted diseases (STDs), and drug use. Since 1991, AIDS cases have also increased by $22 \%$ in people over 50 years of age. AIDS continues to affect those most disenfranchised in US society-the poor and the homeless. While the epidemic has stabilized 
among older white homosexuals, at the same time it is increasing among younger men who have sex with men. We have had great success in reducing HIV transmission among recipients of blood and blood products, and the risk of transmission from transfusion has been reduced by $>99.9 \%$ between 1983 and 1997. And we are making significant strides, thanks to the implementation of therapeutic regimens, in reducing perinatal transmission in this and other developed countries.

These changes in the epidemic demand careful planning, since different prevention and intervention strategies must be applied to each subepidemic. This profile of AIDS epidemiology indicates some of the points of attack both for treatment and prevention. It is instructive to review each of these and reflect on past accomplishments and future challenges that highlight both established research contributions and the daunting challenges ahead.

\section{Recent successes but major problems}

The most dramatic development in the last two years has been the introduction of highly active antiretroviral treatment (HAART), combinations of therapy that include protease inhibitors. This treatment has reduced viral burdens to minimal levels, arrested the progression to AIDS, sharply diminished opportunistic infections and malignancies, and reduced death rates. However, many problems remain. There are many for whom the new drug regimens have not been effective or for whom the side-effects are not tolerable. We do not know how long the benefit of HAART will last, a substantial proportion of patients cannot maintain long-term compliance to drug regimens in spite of effective therapeutic responses, and drug-resistant viral mutants are beginning to emerge in some instances. We are now seeing complications of long-term therapy such as the metabolic complications associated with protease inhibitors. And many patients, particularly in our minority communities, simply do not have the means to access these life-extending therapies. Clearly, there is a need for simpler, less toxic, and cheaper drug regimens, and for the development of new generations of antiviral drugs directed against different viral proteins such as integrase or nucleocapsid. Patients who respond well to HAART do not regain effective anti-HIV immune defenses even after years of viral suppression, although they do regain the ability to contain many opportunistic microbes. An explanation of this immunologic phenomenon is needed, together with creative approaches to reconstitute effective anti-HIV responses. Each of these problems poses an urgent challenge for ongoing research.

Great challenges lie before us in our quest to develop methods to reduce the transmission of infection. Since it is vastly easier and more cost effective to prevent infection rather than treat it, this is a goal of paramount importance. Many points in the transmission cycle are vulnerable to attack.

\section{Perinatal transmission}

Important advances have been made in the diagnosis and prevention of perinatal HIV infection. An NIH-funded clinical trial (ACTG 076) showed that therapeutic intervention protocols can reduce perinatal transmission by $\sim 70 \%$ in industrialized countries where AZT can be made available. In addition, a recent trial of a modified much less expensive protocol for potential use in developing countries has demonstrated the ability to reduce transmission by $50 \%$. To reduce transmission further, additional research is necessary, including studies to understand the timing, mechanisms, and risk factors of perinatal transmission; whether specific HIV strains are more likely to be transmitted; the potential benefit of Caesarean section; and development of newer therapeutic regimens and immunotherapy. The virtual elimination of perinatal transmission in our nation is a goal that should be vigorously pursued.

\section{Women and AIDS}

In view of the dramatic increases in HIV transmission to women-a 4-fold rise in AIDS cases in females over the last 5 years compared to a 2 -fold increase in males- further basic biomedical research related to the etiology and pathogenesis of HIV disease in women is required. Examples include characterizing the cells susceptible to infection in the female reproductive tract, assessing the influence of hormonal modulation on viral susceptibility, and defining the mechanisms of mucosal and vaginal immunity. Although male condom use has been established as an effective method to reduce HIV transmission, we lack effective, inexpensive, and acceptable female-controlled methods to block transmission. There is still no satisfactory vaginal microbicide. STDs, other than AIDS, have been shown to significantly contribute to HIV transmission, and we have yet to control these infections on a global basis. STD prevention and treatment should be an integral component of the AIDS research agenda. Also needed are studies of HIV-related gynecological complications, such as cervical dysplasia, pelvic inflammatory disease, genital ulcers associated with HIV and other STDs.

\section{Drug and alcohol-associated transmission}

HIV transmission among drug users is one of the fastest growing components of the AIDS epidemic in the United States and in many other countries. Until the use of drugs and other substances is effectively reduced, HIV transmission will continue to plague society. Extensive research is needed to understand the disease of addiction itself as well as the complex interaction of alcohol use, drug use, and poor impulse control. Meanwhile, intermediate interventions such as needle exchange have been shown to be partially effective in the reduction of transmission while not increasing the use of injectable drugs. However, the political and social barriers to needle exchange have made it difficult to implement such programs. Continuing efforts are needed to develop societally-acceptable programs for effective control of HIV transmission among drug users.

\section{The behavioral and social sciences}

There has been an impressive effort to study the behaviors that influence the risk of HIV transmission. At their most successful, prevention programs based on these studies have altered sexual and drug using behaviors and reduced the risk of transmission in some instances, but we are still far from realizing the full potential of such prevention research. Furthermore, in all societies, social, ethnic, religious and cultural norms must be considered in the development of any such intervention programs. Understanding these factors, particularly in minority communities, will have an enormous influence on the successful implementation of all potentially effective preventive or therapeutic interventions. Such interventions include diagnostic testing and counselling, case and contact reporting, treatment seeking and adherence to therapy, needle exchange, breast feeding, and 


\section{VACCINE DEVELOPMENT PROGRAM}

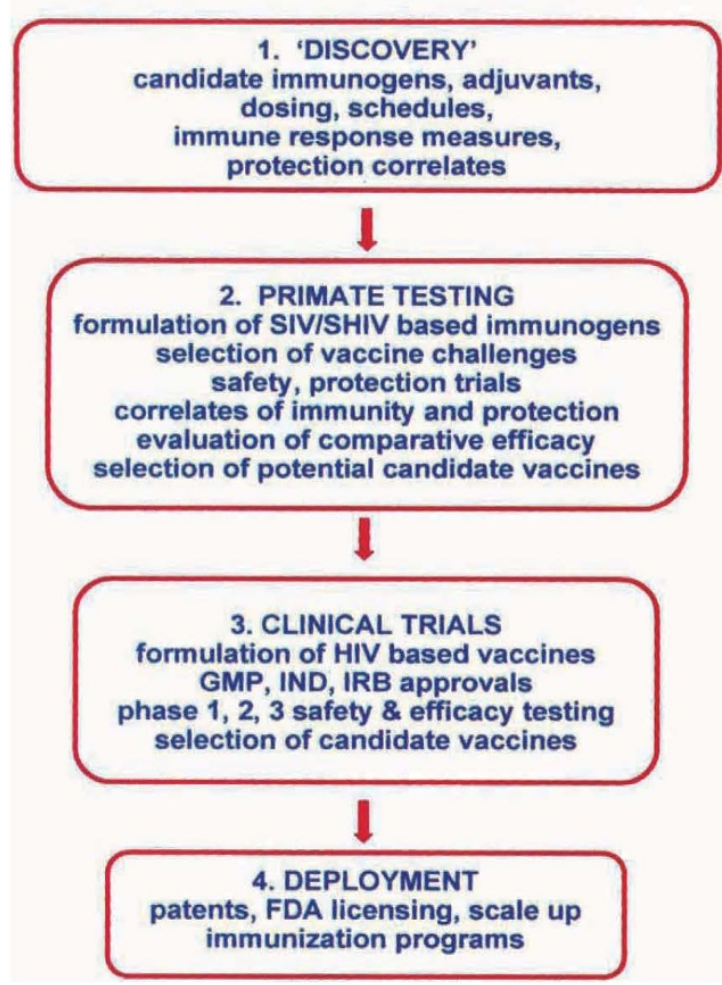

Fig. 2 A possible stepwise program for the development of an AIDS vaccine.

the like. Clearly, the AIDS epidemic offers a continuing challenge to behavioral and social scientists, and their research must be vigorously supported.

\section{Vaccines}

By universal consensus, a safe and effective vaccine is the most important missing element in our armamentarium for the control of AIDS. Recent experience has shown that development of a vaccine is a complex research challenge, because $\mathrm{HIV}$ is unusually well equipped to elude immune defenses, as exemplified by its ability to persist in almost all instances and eventually overcome the immune system. Many successful vaccines depend upon their ability to induce neutralizing antibodies, but neutralization of HIV is peculiarly difficult, perhaps because domains on the envelope protein that bind to receptors are shielded by carbohydrate side chains or are only transiently accessible during viral binding to host cells. Unfortunately, the initial attempts to formulate a vaccine, using recombinant technology to express envelope glycoproteins, have yielded products that elicit antibody with little neutralizing capacity and do not stimulate significant cytolytic T lymphocyte activity. More promising from an efficacy viewpoint are attenuated deletion mutants of SIV, the simian counterpart of HIV. However, the first generation of attenuated mutants that replicate vigorously enough in animals to provide impressive protection also retain residual virulence. Thus, equivalently attenuated HIV cannot be considered safe for human trials.

It is proving more difficult to design an AIDS vaccine than vaccines against other viral diseases. We need a multistage vaccine development program, perhaps along the lines sketched in Figure 2 . This program would begin with an attempt to unravel a wide variety of questions about the structure of the virus, its immunogenicity, the protective role of different components of the immune response, the mechanism of viral escape from immune surveillance, and the like. In addition, fundamental work must be done to develop and refine a number of potentially effective methods for presentation of HIV antigens, including vectors engineered from a wide variety of viruses, bacteria, and naked DNA itself. Building on this base, it will be necessary to use primate models to elucidate the mechanisms of protective immunity and to screen a multitude of candidate immunogens for the most promising products for trial in humans. There are already sufficient partial successes to suggest that we will meet this challenge eventually if we can deploy the full resources of our scientific establishment to attack the problem in an energetic and tenacious manner.

\section{Basic science and investigator driven research}

Although not the focus of this discussion, it is important to emphasize the vital role of basic biomedical research. The research programs of the NIH have always supported a broad and vigorous program to study life processes, using cutting edge methods and a wide range of organisms. Such research, driven by curiosity and initiated by investigators, has provided the constantly advancing knowledge base that permits the development of new applications for the prevention and treatment of disease. Long before the discovery of the first human retrovirus, research on similar viruses of other animals was actively supported, including work on a once obscure subgroup now known as the lentiviruses. This body of research enabled the rapid identification and characterization of HIV, and the study of HIV itself has led to the discovery of several groups of antiviral drugs that have so dramatically changed the outlook for AIDS patients. Consistent with this research vision, a substantial portion of the AIDS budget will continue to be devoted to fundamental biomedical and behavioral research.

The AIDS epidemic, consisting of a number of distinct subepidemics, should be a driving force in the design and implementation of our AIDS research agenda. Each of these subepidemics offers different points of potential attack through various interventions, which in turn require specialized products and programs for treatment or prevention. It is the mandate of the Office of AIDS Research to identify these therapeutic and preventive opportunities and to insure that a diverse and vigorous research program is mounted to meet each of these challenges. Only in that way, can our research agenda be responsive to those who are most in need.

\section{Acknowledgements}

This commentary reflects the contributions of many members of the Office of AIDS Research. The staff of the Division of HIV/AIDS Prevention, CDC, US DHHS, Atlanta, generously provided the data for Figure 1, supplemented with instructive discussions.

\section{Office of AIDS Research, NIH, US DHHS}

Bldg. 31 4C02, 9000 Rockville Pike

Bethesda, MD 20892-2340

email: nathansn@od.nih.gov 